\title{
Opinion on the recent development of environmentally friendly marine anti-fouling coating
}

\author{
WANG Wei \& CAO ZhiQiang* \\ Department of Chemical Engineering and Materials Science, College of Engineering, Wayne State University, 5050 Anthony Wayne Dr, \\ Detroit, Michigan 48202, USA
}

Received October 20, 2016; accepted November 10, 2016; published online November 15, 2016

\begin{abstract}
Marine biofouling is a global problem that is detrimental to both moving ships and static underwater devices. Marine microorganisms tend to attach to any unprotected surface and grow into biofilm, which can be hardly removed even under high shear flow condition [1]. With the long-term accumulation of marine organisms, ships suffer significantly from the increase on the net weight as well as the drag when cruising. Increased drag causes fuel power penalties of up to $86 \%$ at cruising speed; it is notable even a very thin layer of diatom slime would result in $10 \%-16 \%$ penalty [2]. Billions of dollars have been spent globally each year to remove biofouling on ship hulls and marine devices [1]. In addition, increased fuel consumption brings up concerns on the emission of greenhouse gas. Therefore, preventing the biofouling is beneficial in both economic and environmental aspects.

As early as the 1990s, the first generation of anti-fouling coating started to be extensively applied in the marine industry [3]. The paints actively released a broad-spectrum biocide, tributyltin (TBT) that killed most of the potential fouling organisms. Soon it was realized that the long-term release of toxic TBT would cause severe damage to aquatic life, which was responsible for more than $70 \%$ of oxygen production on earth. In 2008, the TBT was banned globally by the international convention. Both $\mathrm{ZnPT}$ and $\mathrm{CuPT}$ have been used to replace TBT, however, there was toxicity concern that they still endangered the life circles in the sea world [1]. Consequently, researchers and companies turned to environment-friendly solutions to the biofouling issue.
\end{abstract}

Corresponding author (email: zcao@wayne.edu)
To limit the environmental impact of biocides, the non-released biocide-containing coating was developed to kill or degrade biofouling in-situ when the microorganisms were attached on the coating. Cationic polymer coating was known for its bactericidal activity by disrupting the bacteria cellular membranes [4]. Nevertheless, such beneficial effect won't last after the bactericidal capability was saturated with an excess amount of microbes. Then biofilm formation would prevail. In addition to biocides, natural enzymes that could degrade or disrupt the biofilm matrix were also incorporated into the marine antifouling coatings [5]. These coatings were found to effectively resist the fouling from specific marine species when corresponding enzyme was incorporated [5]. This technology was thus limited since it was tough to find a broad-spectrum enzyme that was able to degrade biofilms of all marine species. As an additional challenge, the enzyme's stability was difficult to maintain when it was incorporated into the coating.

Rather than to kill the marine organisms, environmentally friendly anti-fouling coatings were designed either to resist the adhesion of biofouling or to make the removal of biofouling easier. Both fouling resist and fouling release strategies are expected to obtain the final marine surface clean and free of biofouling. In recent years, fouling release coatings were developed based on several mechanisms. Stimuli-responsive fouling release coatings utilized functional polymers or nanoparticles that were able to alter their chemical or physical properties in response to changes in environmental $\mathrm{pH}$, temperature, salinity or UV intensity, which resulted in a change of surface property and the release of the adhered biofoulings [3]. Applying these coatings to ship hulls, how- 
ever, may be restricted by the realistically of stimuli changes and effectiveness of fouling release at practical ship cruising conditions. Super-hydrophobic fouling release coatings were studied intensely and had been adopted by ship hulls [6]. Biofouling that attached to super-hydrophobic surfaces would be easily removed due to their low surface tension. On fast moving ships (cruising at 15 knots or above), the loosely-bound biofouling would be wiped off by the shear stress [2]. This property was also called self-cleaning ability. The super-hydrophobic coatings, however, were not suitable for ships that stay for a long period of time or static underwater marine devices.

Compared with fouling release mechanism, fouling resist coatings relied more on the resisting function and performance. Recent studies focused on hydrophilic polymer coatings which attracted a dense layer of water on the surfaces with low polymer-water interfacial energies. When biofouling approached the coating surface, strong hydration increased the entropy penalty which made the surface water molecules difficult to be replaced by biofouling components. This is the mechanism for the hydrophilic coatings to resist the adhesion of marine biofouling [7]. Polyethylene glycol (PEG) is a water-soluble polymer, and polymers based on PEG had been intensely studied as fouling-resist coating materials. Despite their effectiveness to resist fouling from various types of marine microorganisms [8], these PEG-based materials brought up the concerns for long-term functioning, since they were subjective to degradation in the marine environment where abundant oxygen and transition metal ions were presented. Natural hydrophilic polymers, including polysaccharides alginic acid (AA), hyaluronic acid (HA) and pectic acid (PA), were potential candidates as fouling-resist coatings. These natural polymer coatings were found to effectively resist the protein binding, and hematopoietic cell adhesion [9]. However, their resisting capability was not pronounced to most marine organisms. It has been shown that in presence of divalent ions, such as calcium and magnesium, in the marine environment, the polysaccharides tended to be crosslinked and lost their fouling-resisting function [10].

Zwitterionic polymers emerged in recent years as a novel platform to build fouling-resist coating layers for both medical devices and marine coating applications. With an equal amount of positive and negative charges on each repeating unit, the zwitterionic polymer is super-hydrophilic and provides the strongest hydration among hydrophilic polymers [7]. Protein absorption study using Surface Plasma Resonance (SPR) sensor revealed that zwitterionic polymer coating was able to reduce non-specific protein binding to as low as $0.3 \mathrm{ng} / \mathrm{cm}^{2}$, which is the detection limit of the sensor. In addition, glass substrates grafted with zwitterionic polymers were able to greatly reduce the adhesion of ULVA zoospore and diatom [11]. Tracking cyprid behavior on zwitterionic surfaces indicated that the marine organism disliked settling on the super-hydrophilic surface [12]. Despite the advantages of resisting biofouling, nearly all hydrophilic coatings suffered from the instability in an aqueous environment. The hydrophilic polymers tended to dissolve in water rather than staying on the hydrophobic substrates, exposing the unprotected substrates to the marine fouling organisms, particularly at severe shear flow conditions [13].

Besides chemically modifying a surface with a coating, the surface property can also be physically altered by creating micropatterns. Inspired by the marine creatures such as sharks and whales, researchers fabricated micropatterns to mimic the surface topography of their skins and found a reduced biofouling with the new surfaces compared with unmodified substrates [6]. Bio-inspired topography technology requires further study to elucidate whether the pattern alone will be sufficient to achieve the desired antifouling performance. It is reasonable to expect that the topography technology combined with fouling-resist or fouling-release coating materials will further enhance the antifouling function.

In summary, we presented the current approaches to developing environmentally friendly marine anti-fouling coatings and discussed the pros and cons of each of the strategies. For ship hulls and marine devices, the fabrication of effective and durable fouling-resist coatings is highly promising to meet the practical antifouling needs. This challenging task requires future innovative approaches to resolve the long-term reliability issue and mechanical vulnerability of hydrophilic polymer coatings. Bio-inspired topography may also be incorporated into the hydrophilic coatings to further increase the fouling-resist capability.

This work was supported by the U.S. National Science Foundation (Grant No. DMR-1410853), and the faculty start-up fund at Wayne State University, Chemical Engineering and Materials Science.

1 Ciriminna R, Bright F V, Pagliaro M. Ecofriendly antifouling marine coatings. ACS Sustain Chem Eng, 2015, 3: 559-565

2 Callow J A, Callow M E. Trends in the development of environmentally friendly fouling-resistant marine coatings. Nat Commun, 2011, 2: 244-254

3 Nir S, Reches M. Bio-inspired antifouling approaches: The quest towards non-toxic and non-biocidal materials. Curr Opin Biotech, 2016, 39: 48-55

4 Li P, Poon Y F, Li W, et al. A polycationic antimicrobial and biocompatible hydrogel with microbe membrane suctioning ability. Nat Mater, 2011, 10: 149-156

5 Tasso M, Conlan S L, Clare A S, et al. Active enzyme nanocoatings affect settlement of Balanus amphitrite barnacle cyprids. Adv Funct Mater, 2012, 22: 39-47

6 Ferrari M, Benedetti A, Santini E, et al. Biofouling control by superhydrophobic surfaces in shallow euphotic seawater. Colloid Surface A, 2015, 480: 369-375

7 Yang W J, Neoh K G, Kang E T, et al. Polymer brush coatings for combating marine biofouling. Prog Polymer Sci, 2014, 39: 
1017-1042

8 Banerjee I, Pangule R C, Kane R S. Antifouling coatings: Recent developments in the design of surfaces that prevent fouling by proteins, bacteria, and marine organisms. Adv Mater, 2011, 23: 690-718

9 Fyrner T, Lee H H, Mangone A, et al. Saccharide-functionalized alkanethiols for fouling-resistant self-assembled monolayers: Synthesis, monolayer properties, and antifouling behavior. Langmuir, 2011, 27: 15034-15047

10 De Kerchove A J, Elimelech M. Calcium and magnesium cations enhance the adhesion of motile and nonmotile Pseudomonasaeruginosa on alginate films. Langmuir, 2008, 24: 3392-3399

11 Zhang Z, Finlay J A, Wang L, et al. Polysulfobetaine-grafted surfaces as environmentally benign ultralow fouling marine coatings. Langmuir, 2009, 25: 13516-13521

12 Aldred N, Li G, Gao Y, et al. Modulation of barnacle (Balanus amphitrite Darwin) cyprid settlement behavior by sulfobetaine and carboxybetaine methacrylate polymer coatings. Biofouling, 2010, 26: 673-683

13 Wang W, Lu Y, Xie J, et al. A zwitterionic macro-crosslinker for durable non-fouling coatings. Chem Commun, 2016, 52: 4671-4674 